\section{ORIGINAL RESEARCH}

N. Lummel

V. Schoepf

M. Burke

H. Brueckmann

J. Linn

\title{
3D Fluid-Attenuated Inversion Recovery Imaging: Reduced CSF Artifacts and Enhanced Sensitivity and Specificity for Subarachnoid Hemorrhage
}

\author{
BACKGROUND AND PURPOSE: FLAIR images are highly sensitive for SAH. However, CSF flow artifacts \\ caused by conventional FLAIR can produce false-positive results. Here, we compare 3D and 3D FLAIR \\ sequences, focusing on their potential for containing these artifacts and their sensitivity and specificity \\ for detection of SAHs.
}

\begin{abstract}
MATERIALS AND METHODS: We evaluated the following 4 FLAIR sequences: 1) 2D FLAIR at 1.5T, 2) 2D FLAIR, 3) 2D PROPELLER-FLAIR, and 4) 3D Cube-FLAIR at 3T. All sequences were performed in 5 healthy volunteers; sequences 2 and 4 were also performed under routine conditions in 10 patients with focal epilepsy and in 10 patients with SAH. Two neuroradiologists independently conducted the analysis. The presence of flow artifacts in the ventricles and cisterns of healthy volunteers and patients with epilepsy was evaluated and scored on a 4-point scale. Mean values were calculated and compared by using paired $t$ tests. Sensitivity and specificity for SAH detection in sequences 2 and 4 were determined.
\end{abstract}

RESULTS: Cube-FLAIR showed almost no CSF artifacts in the volunteers and the patients with epilepsy; therefore, it was superior to any other FLAIR $(P<.001)$. Sensitivity and specificity of SAH detection by 3 T FLAIR were $58.3 \%$ and $89.4 \%$, respectively, whereas Cube-FLAIR had a sensitivity of $95 \%$ and a specificity of $100 \%$.

CONCLUSIONS: Cube-FLAIR allows FLAIR imaging with almost no CSF artifacts and is, thus, particularly useful for SAH detection.

ABBREVIATIONS: $\mathrm{Cl}=$ confidence interval; $\mathrm{GRE}=$ gradient-recalled echo; PROPELLER $=$ periodically rotated overlapping parallel lines with enhanced reconstruction; SE = standard error

$\mathbf{F}$ AIR is a valuable MR imaging technique for the detection of intracranial hemorrhage, including SAH and intraventricular hemorrhage. ${ }^{1,2}$ However, one of the major limitations of FLAIR imaging is the presence of CSF artifacts. ${ }^{3}$ These artifacts can compromise the sensitivity and specificity of FLAIR by contributing to false-negative or false-positive interpretations of abnormalities in the CSF space (eg, in the detection of SAH or intraventricular hemorrhage). ${ }^{1,4,5}$

Therefore, significant work has been conducted to reduce CSF artifacts in 2D FLAIR images. ${ }^{6-12}$ Recently, 3D FLAIR sequences were developed. Initial reports by using this $3 \mathrm{D}$ technique noted a significant reduction in the number of highsignal intensity artifacts from the inflow of noninverted CSF and from pulsatile motion compared with 2D FLAIR. ${ }^{13-15}$

In this study, we compared the likelihood of producing CSF flow artifacts in 4 different FLAIR sequences: standard 2D FLAIR performed at 1.5T (1.5T FLAIR), standard 2D FLAIR (3T FLAIR), PROPELLER-FLAIR, and 3D Cube-FLAIR performed at 3T. Additionally, we compared the sensitivity and specificity of Cube-FLAIR with that of standard 2D FLAIR for the detection of SAHs at 3T.

Received January 13, 2011; accepted after revision April 4.

From the Department of Neuroradiology (N.L., H.B., J.L.), University of Munich, Munich, Germany; Division of Neuro- and Musculoskeletal Radiology (V.S.), Department of Radiology and the MR Centre of Excellence (V.S.), Medical University Vienna, Vienna, Austria; and GE Healthcare (M.B.), Solingen, Germany.

Please address correspondence to Nina Lummel, MD, Department of Neuroradiology, University of Munich, Marchioninistr 15, 81377 Munich, Germany; e-mail: nina.lummel@ med.uni-muenchen.de

Indicates article with supplemental on-line tables.

http://dx.doi.org/10.3174/ajnr.A2682

\section{Materials and Methods}

This study was approved by our institutional review board. Informed consent was obtained from all patients who participated in the MR imaging investigations.

\section{Data Acquisition and Analysis}

The parameters for the 4 different FLAIR sequences are detailed in On-line Table 1. TR, TE, and TI of the 4 sequences were optimized before the beginning of the study to obtain a good and comparable gray and white matter contrast. Sequence 1 was acquired on a $1.5 \mathrm{~T}$ scanner (Magnetom Symphony; Siemens Medical Solutions, Erlangen, Germany) with a standard head coil, while sequences 2-4 were performed on a 3T scanner (Signa HDxt; GE Healthcare, Milwaukee, Wisconsin) with an 8-channel head coil. Parallel imaging was applied in the acquisition of the Cube-FLAIR sequence to reduce scanning time. Cube-FLAIR is a 3D fast-spin-echo sequence with inversion recovery preparation that uses variable refocusing flip angles to establish a pseudo-steady-state condition in which relaxation is counterbalanced. Relaxation counterbalancing results in reduced or even halted signal intensity decay during long echo trains and lacks image blurring. ${ }^{16}$ Cube-FLAIR isotropic voxel size allows arbitrary multiplanar reconstructions of the sagittal source images.

The study comprised 3 parts:

1. Comparison of the different FLAIR sequences with a focus on the presence of CSF flow artifacts in healthy volunteers.

To compare the 4 sequences for their likelihood of generating artifacts, we performed all 4 FLAIR sequences on 5 healthy volunteers ( 2 men; mean age, 27 years, range, $22-33$ years).

2. Comparison of 3D FLAIR and conventional 2D FLAIR at 3T under routine conditions.

To evaluate the potential of Cube-FLAIR under more clinically rele- 
vant conditions, we performed Cube-FLAIR and standard 3T FLAIR on 10 consecutive patients with focal seizures ( 5 men; mean age, 37.5 years, range, 20-53 years) during routine diagnostic work-up.

3. Sensitivity and specificity of 3T FLAIR and Cube-FLAIR for SAH.

To determine the sensitivity and specificity of Cube-FLAIR versus standard 2D FLAIR for the detection of SAH at 3T, we performed both sequences on 10 consecutive patients with noncontrast CTproved SAH (5 men; mean age, 57.6 years, range, 34-74 years; Online Table 2). The FLAIR datasets of 10 patients with focal epilepsy (6 men; mean age, 37.8 years, range, $18-56$ years) were used as controls. To avoid recognition effects, we used datasets from patients with epilepsy that were not previously used in part 2 of the study.

\section{Image Interpretation and Statistical Data Analysis}

For data analysis, the sagittal Cube-FLAIR images were reformatted into 5-mm-thick axial sections to match the section thickness of standard 2D FLAIR. Two experienced neuroradiologists who were blinded to all patient identification and clinical information independently analyzed all FLAIR datasets on a standard PACS workstation (MagicView VE 42; Siemens, Erlangen, Germany). The datasets were presented to the readers in a pseudorandomized order.

\section{Study Parts 1 and 2}

All FLAIR datasets acquired in parts 1 and 2 of the study were evaluated for the presence of artifacts in the lateral, third, and fourth ventricles as well as in the suprasellar, perimesencephalic, prepontine, and perimedullary cisterns by using a 4-point scale modified according to the one proposed by Chagla et $\mathrm{al}^{15}: 0=$ no pulsation artifacts, $1=$ minimal pulsation artifacts, $2=$ moderate pulsation artifacts, and $3=$ severe pulsation artifacts that obscure adjacent structures.

Statistical analyses were performed by using the Statistical Package for the Social Sciences, Version 17.0 (SPSS, Chicago, Illinois). To access differences in artifacts proneness between the different FLAIR sequences, we submitted artifacts scorings to repeated ANOVAs. Sphericity was analyzed by using the Mauchly test. Sphericity relates to the equality of variances of the differences between levels of the factors for the repeated measures. Sphericity requires that the variances for each dataset be equal, which is an assumption of the repeated-measures ANOVA. The repeated measures in this setting were the different sequences; therefore, sphericity was tested for variances between the different measurement parameters. Agreement between the 2 raters was tested by using the Cohen $\kappa$. Afterward paired-samples $t$ tests were applied to test for significant differences in the presence of CSF artifacts in the 4 FLAIR sequences acquired in the healthy volunteers. Analogously, a paired-samples Student $t$ test was used to compare the axial 3T FLAIR and Cube-FLAIR in the patients with epilepsy. The $\alpha$ level for all tests was set at $P=.05$.

\section{Study Part 3}

To determine the sensitivity and specificity of Cube-FLAIR versus standard 3T FLAIR for SAH detection, the readers noted the following: 1) the overall presence or absence of an SAH in each patient, and 2) the distribution of the SAH in the respective patient. We considered the following locations (CSF compartments): hemispheric cortical sulci; lateral, third, and fourth ventricles; and suprasellar, perimesencephalic, prepontine, and perimedullary cisterns. The diagnostic confidence was rated on a 5 -point scale $(5=$ absolutely certain, $4=$ very certain, $3=$ certain, $2=$ not very certain, $1=$ uncertain).
The reference standard for the presence and extent of an SAH was based on the findings in NCCT examinations, which were analyzed by the same 2 neuroradiologists in consensus. Furthermore, CSF was positive for SAH in all patients.

Sensitivity and specificity parameters of the 2 different FLAIR sequences for the overall presence of a SAH and for the involvement of 3 locations (sulci, ventricles, and cisterns), pooled from the results of the evaluation on a per-location basis, were calculated. Furthermore, inter-rater reliability analysis by Cohen $\kappa$ statistics was performed to determine consistency among raters.

\section{Results}

Study Parts 1 and 2: Presence of CSF Artifacts

ANOVA analysis of the 4 sequences performed in the volunteers revealed significant differences $(P<.001)$. Mauchly test indicated that the assumption of sphericity was violated $\left(\chi^{2}\right.$ [27] $=44.47, P=.02$ ). Thus, results were corrected by using Greenhouse-Geisser estimates of sphericity $(\varepsilon=0.6)$. The mean artifacts score was 1.95 for 1.5 T FLAIR (95\% CI, $1.56-$ 2.34; SE, 0.18), 1.6 for 3T FLAIR (95\% CI, 1.21-1.99; SE, 0.18), 1.25 for PROPELLER-FLAIR (95\% CI, 0.86-1.64; SE, 0.18), and 0.025 for Cube-FLAIR (95\% CI, 0.36-0.41; SE, 0.18). Pair-wise comparison of the sequences indicated that there were significantly fewer CSF artifacts on Cube-FLAIR compared with any of the other 3 FLAIR sequences $(P<.001$, Figs 1 and 2).

The inter-rater agreement was consistent for all sequences in study part $1 \kappa(1.5 \mathrm{~T}$ FLAIR $)=0.963,(95 \% \mathrm{CI}, 0.89-1$; SE, $0.036) ; \kappa(3$ T FLAIR $)=0.926(95 \% \mathrm{CI}, 0.83-1 ; \mathrm{SE}, 0.05) ; \kappa$ $($ PROPELLER-FLAIR $)=0.914(95 \%$ CI, $0.80-1 ;$ SE, 0.06$) ; \kappa$ $($ Cube-FLAIR $)=1 .{ }^{17}$

ANOVA for the epilepsy patient group also revealed significant differences between the sequences $(P<.001)$. Mauchly test indicated that the assumption of sphericity was violated $\left(\chi^{2}[27]=51.43, P=.004\right)$. Thus, results were corrected by using Greenhouse-Geisser estimates of sphericity $(\varepsilon=0.56)$. The 2-sample $t$ test again indicated that there were significantly fewer CSF artifacts in Cube-FLAIR compared with the 3T FLAIR $(P<.001$, mean artifacts score for Cube-FLAIR $=$ 0.05 ; mean artifact score for the $3 \mathrm{~T}$ FLAIR $=1.375$; Figs 3 and 4).

The inter-rater agreement was consistent in study part $2(\kappa$ $(3 \mathrm{~T}-\mathrm{FLAIR})=0.963(95 \% \mathrm{CI}, 0.91-1 ; \mathrm{SE}, 0.026) ; \kappa($ CubeFLAIR) $=1)$.

\section{Study Part 3: SAH detection}

NCCT revealed a basal SAH in 6 patients, a basal SAH with intraventricular hemorrhage in 1 patient, and a convexity SAH in 3 patients (On-line Table 2 and Fig 5). The mean time interval between CT and MR imaging was 1.7 days (range, 1-3 days).

Regarding the overall presence of an SAH, the sensitivity of the 3 T FLAIR was $65 \%(95 \% \mathrm{CI}, 40.9 \%-83.7 \%)$ with a specificity of $70 \%$ ( $95 \%$ CI, $45.7 \%-87.2 \%$ ), while the Cube-FLAIR sequence had a sensitivity and specificity of $100 \%$ (95\% CI, $80.0 \%-100 \%)$. For the overall evaluation on a per-location basis, sensitivity and specificity of the 3T FLAIR for SAHs were $58.3 \%$ (95\% CI, 44.9\%-70.7\%) and 89.4\% (95\% CI, 85.5\%$92.4 \%)$, while Cube-FLAIR had a sensitivity of $95.0 \%(95 \%$ 
1.5T FLAIR

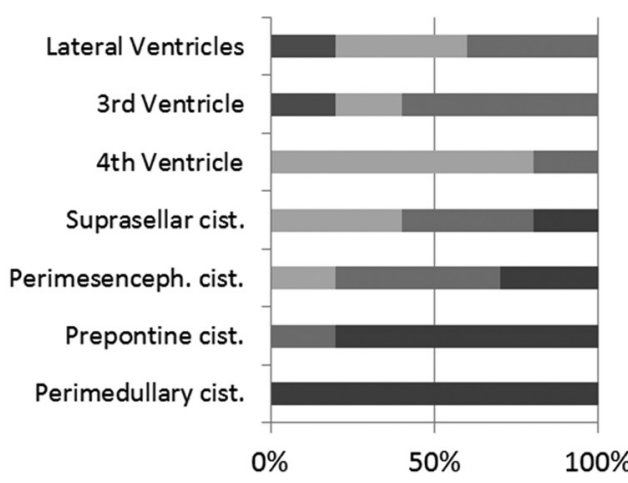

PROPELLER-FLAIR

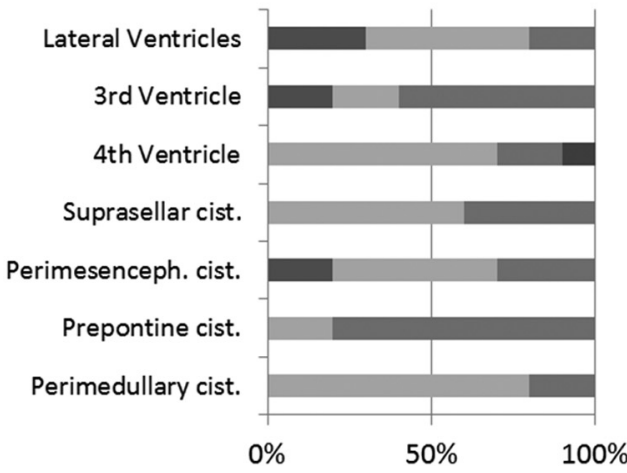

3T FLAIR

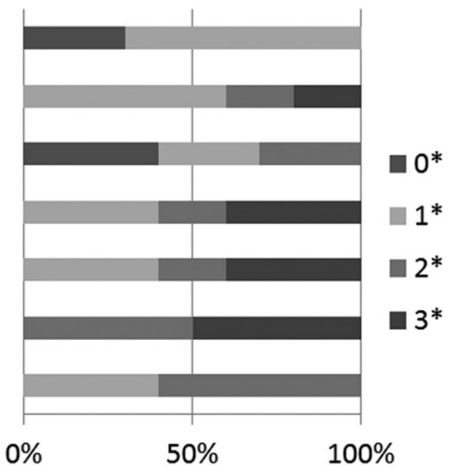

Cube-FLAIR

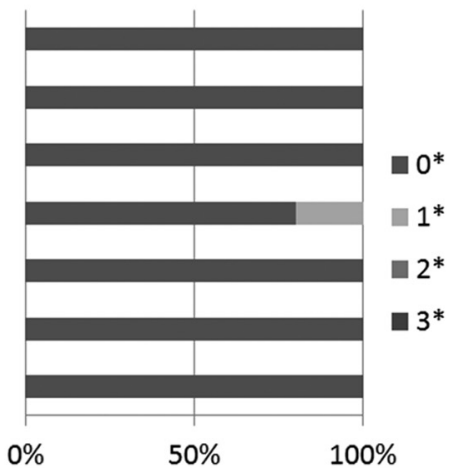

Fig 1. Presence of CSF artifacts in the different FLAIR datasets acquired under study conditions in healthy volunteers. Percentages (asterisks) indicate frequencies of scoring the presence of CSF artifacts in the respective region as no (0), minimal (1), and moderate (2) pulsation artifacts or severe pulsation artifacts that obscure adjacent structures (3).

CI, $85.2 \%-98.7 \%)$ and a specificity of $100 \%$ (95\% CI, $98.6 \%-$ $100 \%$ ) (Table).

Sensitivity of the 3T FLAIR for a sulcal SAH was $85.7 \%$ (95\% CI, 56.2\%-97.5\%) with a specificity of 97\% (95\% CI, $88.5 \%-99.5 \%$ ), while Cube-FLAIR had a sensitivity of $78.6 \%$ (95\% CI, 44.8\%-94.3\%) and a specificity of 100\% (95\% CI, 93.1\%-100\%). 3T FLAIR had a sensitivity and specificity of $100 \%$ (95\% CI, 39.6\%-100\%) and 93.6\% (95\% CI, 88.2\%$96.7 \%)$, respectively, for intraventricular hemorrhage, and a sensitivity and specificity of $45.2 \%$ (95\% CI, 30.2\%-61.2\%) and $79.7 \%$ (95\% CI, 71.1\%-86.3\%), respectively, for cisternal SAH. The sensitivity and specificity of Cube-FLAIR was $100 \%$ for both intraventricular hemorrhage (sensitivity: 95\% CI, 39.6\%-100\%; specificity: 95\% CI, 97.0\%-100\%) and cisternal SAH (sensitivity: 95\% CI, 89.6\%-100\%; specificity: 95\% CI, 96.1\%-100\%).

The inter-rater agreement for the presence of SAH on a per-location basis was moderate for 3T FLAIR $(\kappa=0.495$, 95\% CI, 0.35-0.64; SE, 0.075) and outstanding for CubeFLAIR $(\kappa=0.95 ; 95 \%$ CI, 0.85-1; SE, 0.05).

\section{Discussion}

High-signal intensity artifacts within both the SAH and the ventricles are a common phenomenon in FLAIR imaging and are predominately due to CSF flow (CSF flow artifacts) and vascular pulsation (ghosting artifacts). ${ }^{1,18}$ As previously shown, CSF flow artifacts predominantly occur in the basal cisterns and in the third and fourth ventricles ${ }^{5,18}$; they are less common and less severe in the lateral ventricles and over the convexities of the cerebral hemispheres. The increase in sever- ity and frequency of CSF flow artifacts in the basal cisterns and the third and fourth ventricles is most likely multifactorial. ${ }^{18}$ One potential cause of CSF flow artifacts is the reflux of spinal CSF into these ventricles through the posterior fossa. A second factor influencing CSF flow artifacts is the increased velocity of CSF flow through the third and fourth ventricles, increasing the rate of CSF inflow from the lateral ventricles during inversion delay.

The second contributor to high-signal intensity artifacts in the CSF space on axial FLAIR images is the presence of ghosting artifacts, which are caused by the periodic motion of vascular pulsation, in which there is synchrony between the phase-encoding steps and the motion. ${ }^{5}$ In rare instances, these artifacts can be mistaken for hyperintensities in the subarachnoid space on FLAIR images.

Because CSF artifacts can compromise the sensitivity and specificity of FLAIR images in detecting pathologies in the CSF space, there has been much effort to reduce CSF artifacts in standard axial 2D FLAIR images. ${ }^{6-11}$ The most common technique is to widen the inversion pulse to diminish inflow of noninverted magnetization. ${ }^{18-20}$ Other mechanisms that have been used are the following: $k$-space reordering by TI at each section position, ${ }^{7}$ tailored radio-frequency pulses, ${ }^{11}$ increasing the number of interleaving acquisitions, ${ }^{10}$ and adiabatic inversion pulses. ${ }^{6}$ Nevertheless, all of these attempts to eradicate CSF artifacts were not successful.

In 1999, Pipe $^{21}$ proposed a new MR imaging technique called PROPELLER. This technique seeks to reduce artifacts induced by in-plane rotation and translational head motion by using an alternative way of sampling $k$-space. Winter- 

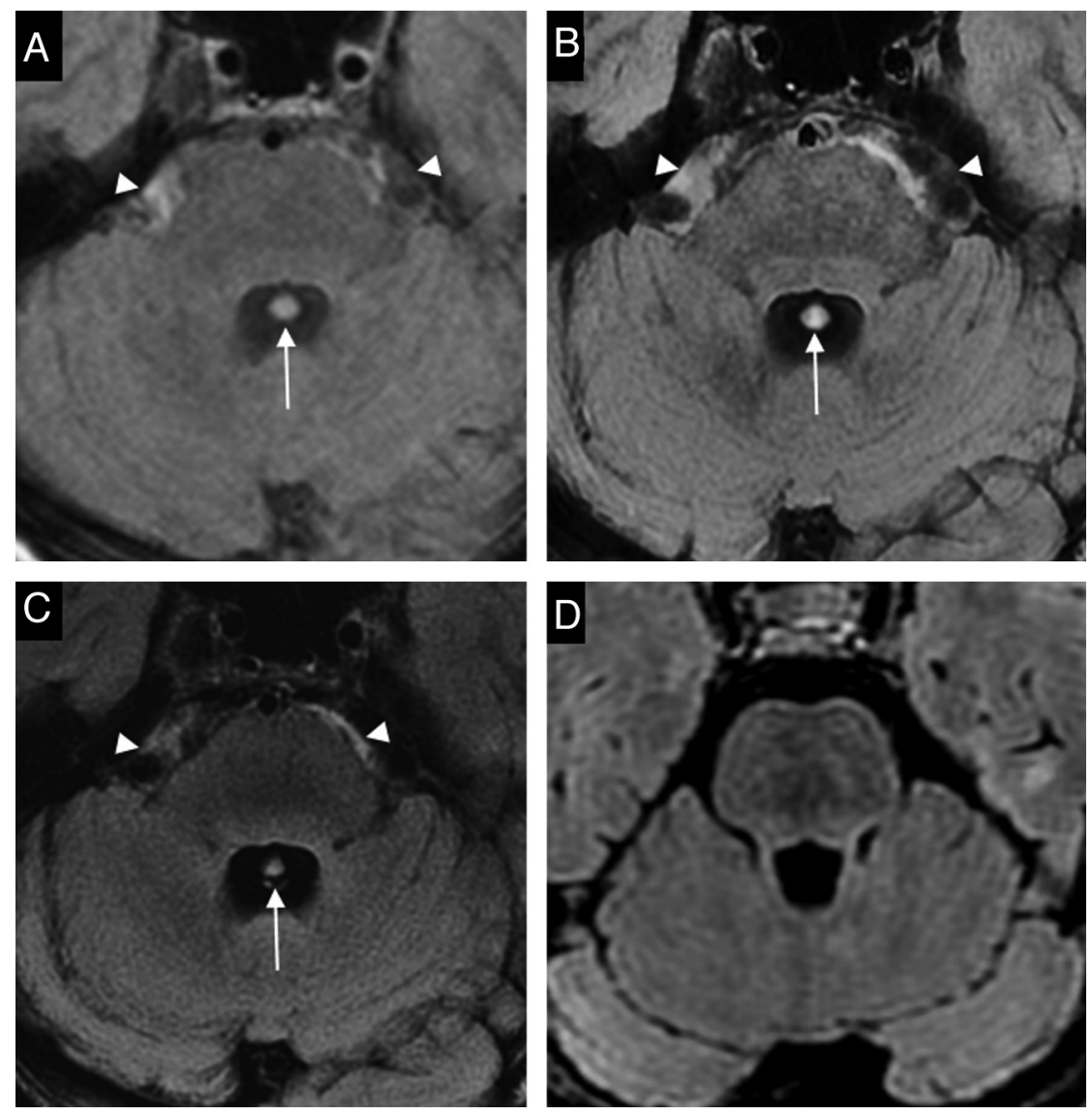

Fig 2. Four different FLAIR images of the same healthy volunteer. Axial standard 2D FLAIR at 1.5T $(A)$, at $3 T(B)$, and PROPELLER FLAIR $(C)$ and axial reconstructions of the 3D Cube-FLAIR (D). CSF artifacts are visible in the fourth ventricle (arrows) and in the prepontine cistern (arrowheads) on all FLAIR-images $(A-C)$ except on the 3D Cube-FLAIR (D).

3T FLAIR

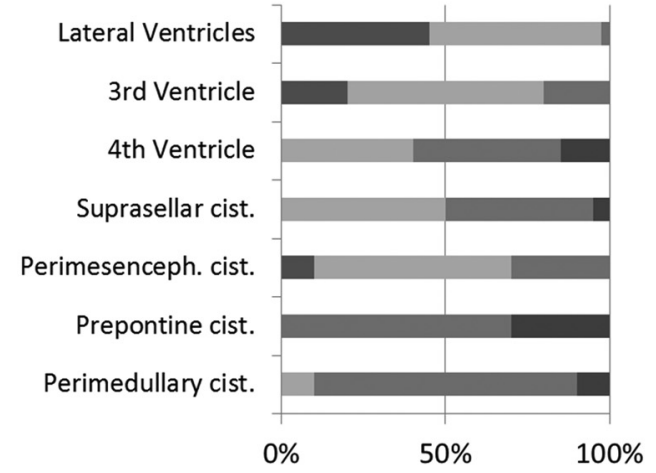

Cube-FLAIR

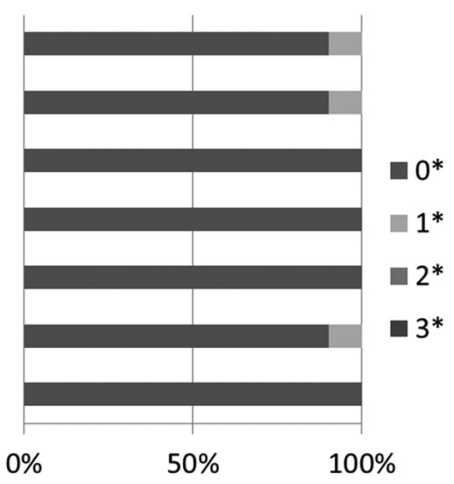

Fig 3. Presence of CSF artifacts in the different FLAIR datasets acquired under routine conditions in patients with epilepsy. Percentages (asterisks) indicate frequencies of scoring the presence of CSF artifacts in the respective region as no (0), minimal (1), and moderate (2) pulsation artifacts or severe pulsation artifacts that obscure adjacent structures. (3).

sperger et al $^{12}$ demonstrated significantly less vascular pulsation, ghosting (motion), and Gibbs artifacts in PROPELLERFLAIR than in standard axial FLAIR at 3T. They analyzed only the fourth ventricle for CSF pulsation artifacts and found flow artifacts in significantly more cases with PROPELLER than with standard FLAIR. In our study, we found that PROPELLER-FLAIR did not significantly reduce CSF flow artifacts within the basal cisterns or ventricles.

The potential for 3D FLAIR techniques to significantly reduce CSF space artifacts has previously been described at $1.5 \mathrm{~T}^{13}$ and $3 \mathrm{~T} .{ }^{14,15}$ Our results further support these findings. We found that while CSF artifacts are essentially nonexistent on Cube-FLAIR sequences, they are prominent on standard 1.5T and 3T FLAIR sequences and on PROPELLER-FLAIR. Our data indicate that Cube-FLAIR could be a useful tool in MR imaging of SAH. Regarding technical aspects, differences of imaging parameters among the 4 FLAIR sequences used in this investigation are conspicuous. These differences are due to the fact that all 4 sequences were optimized before the beginning of the study to obtain a good and comparable gray and white matter contrast. Furthermore, due to the different nature of data acquisition between standard 3T FLAIR and Cube-FLAIR (ie, the extended echo-train length with modulated flip angles), both pulse sequences have been indepen- 

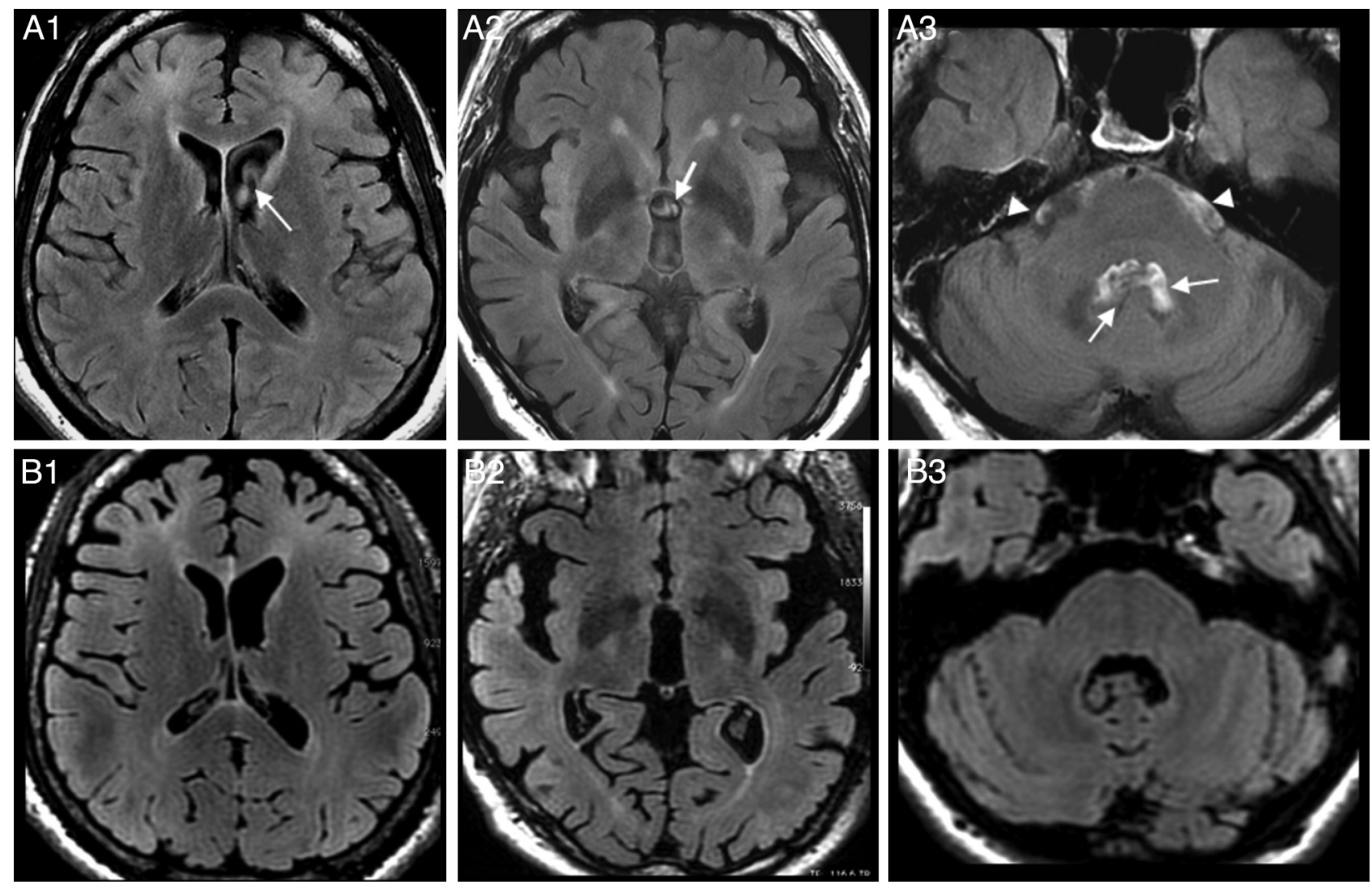

Fig 4. Axial standard $2 D$ FLAIR (A1-3) and axial reconstructions of the 3D Cube-FLAIR (B1-3) acquired in a patient with epilepsy on a 3T scanner. On the 2D FLAIR images, considerable CSF artifacts were present in the left lateral ventricle (arrow in $A 1$, judged as grade 1 by both readers), in the third ventricle (arrow in $A 2$, judged as grade 2), in the fourth ventricle (long arrows in $A 3$, judged as grade 3), and in the prepontine cistern (arrowheads in A3, judged as grade 2). The corresponding sections of the 3D Cube-FLAIR (B1-3) were not affected by these artifacts.

dently optimized to best suppress CSF, and it has been found that Cube-FLAIR best suppresses CSF with a slightly shorter TI than 3T FLAIR. A more technically detailed description of flip angle modulation can be found in Busse et al (2006). ${ }^{16}$ In general, Cube-FLAIR uses much thicker volumes than standard 3T FLAIR to suppress CSF. Cube-FLAIR accomplishes this by inversion recovery preparation such that CSF inflow during $\mathrm{T} 1$ penetrates less of the imaging volume, which results in better suppression of CSF. As a result, fewer flow artifacts are found in Cube-FLAIR imaged volume compared with 3T FLAIR.

In anticipation of potential drawbacks to 3D FLAIR techniques, patient movement and subsequent image degradation during the relatively long imaging time have been addressed. ${ }^{13}$ To test the clinical relevance of this potential disadvantage, we analyzed datasets of patients with focal epilepsy, acquired in the clinical environment. Our MR imaging epilepsy protocol lasts for approximately 31 minutes, and Cube-FLAIR is the last sequence acquired. Even under these conditions, we could confirm the consistency of artifacts eradication on CubeFLAIR. The sequence provides $1.4-\mathrm{mm}$ isotropic images covering the whole brain and allowing multiplanar reconstructions in a scanning time of 6 minutes and 9 seconds, compared with 3 minutes and 58 seconds for the axial 3T FLAIR. Thus, the longer scanning time does not seem to reduce the advantages of the $3 \mathrm{D}$ sequence.

While the available studies concordantly show the value of 3D FLAIR sequences for artifacts reduction in the CSF spaces, data on the clinical relevance of this advantage for detecting pathologies in the subarachnoid space or ventricles have been lacking. While NCCT is still the imaging technique of choice in the emergency setting for most clinical departments, an increasing number of institutions use MR imaging as the initial imaging technique, especially in patients with stroke. It has been shown that the sensitivity of MR imaging, especially by using GRE sequences, for intracerebral hemorrhage is equal to that of NCCT. ${ }^{22}$ Also, the sensitivity of MR imaging for SAH detection is high if FLAIR or GRE sequences are performed. ${ }^{23-25}$ The major limitation of the GRE sequence is that the strong susceptibility artifacts at the skull base cannot be reliably distinguished from low signal intensity due to subarachnoid blood. ${ }^{26}$ The value of conventional FLAIR imaging is limited by its likelihood of generating artifacts within the CSF spaces and the potential for false-positive results. Here, we could demonstrate that - due to the virtual absence of CSF space artifacts - the sensitivity and specificity of Cube-FLAIR for the overall detection of SAHs and for the detection of SAHs in the ventricles and cisterns are significantly superior to those of standard 3T FLAIR. For hemorrhages in the ventricles and cisterns, where most SAHs caused by rupture of aneurysms are found, Cube-FLAIR had a sensitivity and specificity of $100 \%$. False-negative results were only evident in the evaluation of sulcal SAHs.

On the basis of these initial results, we conclude that CubeFLAIR helps to overcome the limitations of an MR imagingbased work-up of SAH. 

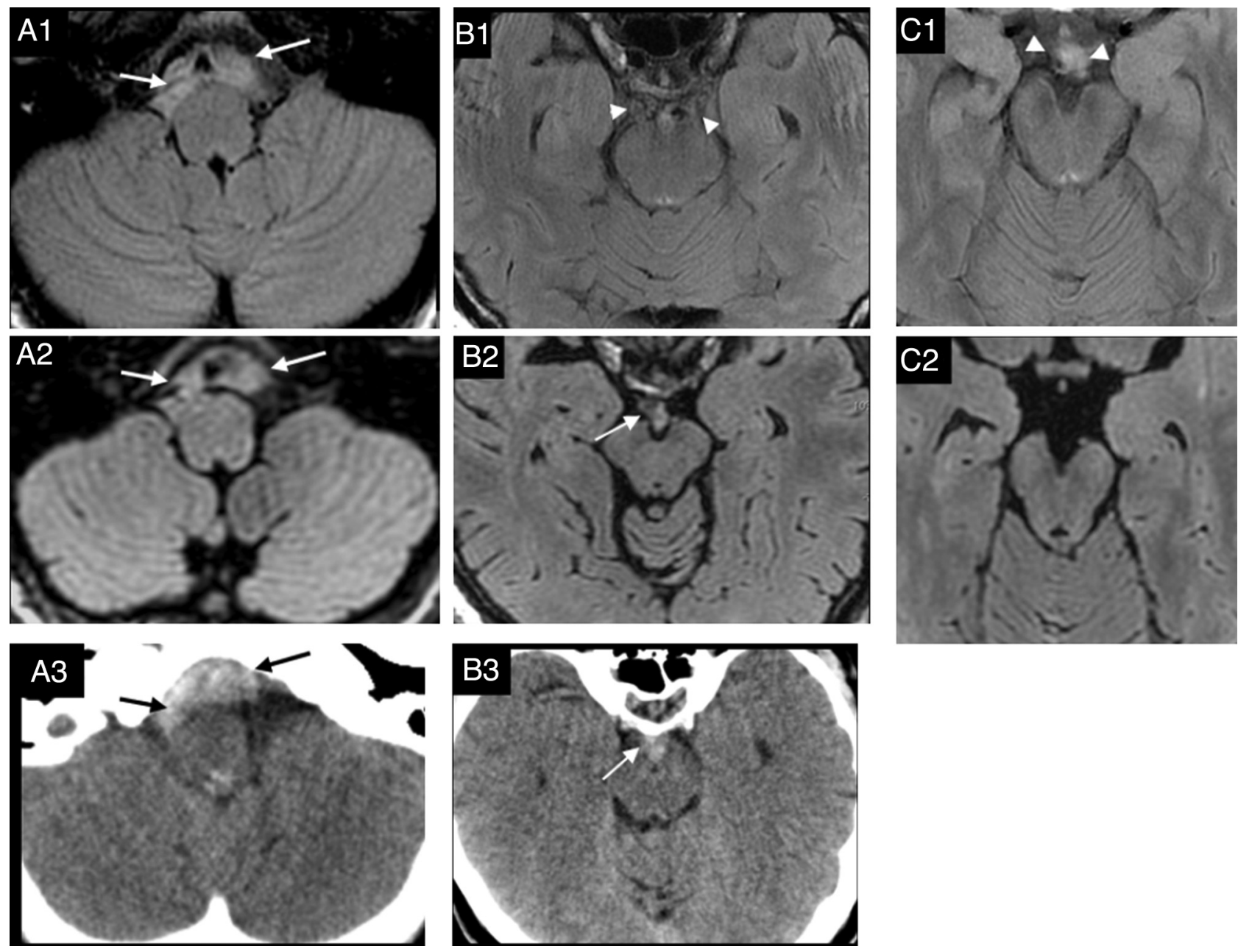

Fig 5. Axial standard 2D FLAIR images $(A 1, B 1, C 1)$, axial reconstructions of the 3D Cube-FLAIR $(A 2, B 2$, C2), and NCCT (A3, B3) of 3 different patients $(A-C)$. $A 1-3$, A 34-year-old patient with CT-proved SAH in the perimedullary cistern (black arrows in A3). SAH is well-delineated on the 2D FLAIR (arrows in A1) and on the Cube-FLAIR (arrows in A2). B1-3, A 53-year-old patient with CT-proved SAH in the interpeduncular fossa (arrow in B3). While the Cube-FLAIR (B2) shows the circumscript SAH (arrow in B2) analogous to the CCT, the SAH can hardly be distinguished from CSF artifacts (arrowheads in B1) on standard 2D FLAIR (B1). C1-2, Control patient with epilepsy but without SAH. The standard 2D FLAIR findings were judged as false-positive with regard to the presence of SAH within the perimesencephalic and suprasellar cisterns by both readers (arrowheads in C1), whereas the Cube-FLAIR (C2) clearly shows the absence of SAH in this location.

\begin{tabular}{|c|c|c|c|c|}
\hline \multicolumn{5}{|c|}{$\begin{array}{l}\text { Contingency table comparing the results of the diagnostic tests (2D } \\
\text { and 3D FLAIR) and the reference standard (CT) for patients with } \\
\text { SAH and controls (patients with epilepsy) }\end{array}$} \\
\hline \multirow[b]{2}{*}{ Diagnostic Test } & \multicolumn{2}{|c|}{ 3T FLAIR } & \multicolumn{2}{|c|}{ Cube-FLAIR } \\
\hline & Positive & Negative & Positive & Negative \\
\hline \multicolumn{5}{|l|}{$\mathrm{A}^{\mathrm{a}}$} \\
\hline Positive & 13 & 7 & 20 & 0 \\
\hline Negative & 6 & 14 & 0 & 20 \\
\hline \multicolumn{5}{|l|}{$\mathrm{B}^{\mathrm{b}}$} \\
\hline Positive & 35 & 25 & 57 & 3 \\
\hline Negative & 36 & 304 & 0 & 340 \\
\hline
\end{tabular}

a Per patient

b Per location.

\section{Limitations}

The small number of patients with $\mathrm{SAH}$ is a limitation of our study. However, due to the often severe clinical symptoms of $\mathrm{SAH}$, our institution does not regularly perform MR imaging on these patients. Thus, we propose that additional studies should confirm our initial findings and test the potential of Cube-FLAIR for other pathologies that have high signal intensity within the CSF space. ${ }^{27-31}$
Similar to authors of previous MR imaging studies on SAH, we used CT as a reference standard to determine the presence and localization of SAHs. ${ }^{2,23-25}$ To further enhance the diagnostic certainty regarding the overall presence of a $\mathrm{SAH}$, we used a positive CSF result as additional inclusion criterion. Yet, it cannot be absolutely excluded that CT yielded falsenegative results in specific locations.

\section{Conclusions}

We showed that Cube-FLAIR was virtually unimpaired by high-signal intensity-artifacts within the CSF space, while these artifacts were prominent in all other tested FLAIR sequences. We found that Cube-FLAIR had a high sensitivity and specificity for the detection of SAHs, indicating that this sequence may help to overcome the most persistent pitfall of standard FLAIR imaging for CSF space diseases, namely CSF artifacts. Further investigation is needed to confirm these initial findings.

Disclosures: Michael Burke, Research Support (including provision of equipment or materials): provision of MR pulse sequence; Details: on the basis of a research agreement, GE 
Healthcare provided an MR pulse sequence prototype, which, in the meantime, is available as product; provision of technical information; discussion of MR imaging technical aspects.

\section{References}

1. Bakshi R, Kamran S, Kinkel PR, et al. Fluid-attenuated inversion-recovery MR imaging in acute and subacute cerebral intraventricular hemorrhage. AJNR Am J Neuroradiol 1999;20:629-36

2. Noguchi K, Ogawa T, Inugami A, et al. Acute subarachnoid hemorrhage: MR imaging with fluid-attenuated inversion recovery pulse sequences. Radiology 1995; 196:773-77

3. Adams JG, Melhem ER. Clinical usefulness of T2-weighted fluid-attenuated inversion recovery MR imaging of the CNS. AJR Am J Roentgenol 1999;172:529-36

4. Lummel N, Wiesmann $\mathrm{M}$, Bruckmann $\mathrm{H}$, et al. The value of different magnetic resonance imaging sequences for the detection of intraventricular hemorrhages. Klin Neuroradiol 2010 Feb 28 [Epub ahead of print]

5. Stuckey SL, Goh TD, Heffernan T, et al. Hyperintensity in the subarachnoid space on FLAIR MRI. AJR Am J Roentgenol 2007;189:913-21

6. Hajnal JV, Oatridge A, Herlihy AH, et al. Reduction of CSF artifacts on FLAIR images by using adiabatic inversion pulses. AJNR Am J Neuroradiol 2001;22:317-22

7. Herlihy AH, Hajnal JV, Curati WL, et al. Reduction of CSF and blood flow artifacts on FLAIR images of the brain with k-space reordered by inversion time at each slice position (KRISP). AJNR Am J Neuroradiol 2001;22:896-904

8. Herlihy AH, Oatridge A, Curati WL, et al. FLAIR imaging using nonselective inversion pulses combined with slice excitation order cycling and $\mathrm{k}$-space reordering to reduce flow artifacts. Magn Reson Med 2001;46:354-64

9. Oatridge A, Curati WL, Herlihy AH, et al. Evaluation of a FLAIR sequence designed to reduce CSF and blood flow artifacts by use of k-space reordered by inversion time at each slice position (KRISP) in high grade gliomas of the brain. J Comput Assist Tomogr 2001;25:251-56

10. Tanaka N, Abe T, Kojima K, et al. Applicability and advantages of flow artifactinsensitive fluid-attenuated inversion-recovery $\mathrm{MR}$ sequences for imaging the posterior fossa. AJNR Am J Neuroradiol 2000;21:1095-98

11. Wu HM, Yousem DM, Chung HW, et al. Influence of imaging parameters on high-intensity cerebrospinal fluid artifacts in fast-FLAIR MR imaging. AJNR Am J Neuroradiol 2002;23:393-99

12. Wintersperger BJ, Runge VM, Biswas J, et al. Brain magnetic resonance imaging at 3 Tesla using BLADE compared with standard rectilinear data sampling. Invest Radiol 2006;41:586-92

13. Kallmes DF, Hui FK, Mugler JP, 3rd. Suppression of cerebrospinal fluid and blood flow artifacts in FLAIR MR imaging with a single-slab three-dimensional pulse sequence: initial experience. Radiology 2001:221:251-55

14. Naganawa S, Koshikawa T, Nakamura T, et al. Comparison of flow artifacts between 2D-FLAIR and 3D-FLAIR sequences at 3 T. Eur Radiol 2004;14:1901-08

15. Chagla GH, Busse RF, Sydnor R, et al. Three-dimensional fluid attenuated inversion recovery imaging with isotropic resolution and nonselective adiabatic inversion provides improved three-dimensional visualization and cere- brospinal fluid suppression compared to two-dimensional flair at 3 Tesla. Invest Radiol 2008;43:547-51

16. Busse RF, Hariharan $\mathrm{H}, \mathrm{Vu}$ A, et al. Fast spin-echo sequences with very long echo trains: design of variable refocusing flip angle schedules and generation of clinical T2 contrast. Magn Reson Med 2006;55:1030-37

17. Landis JR, Koch GG. The measurement of observer agreement for categorical data. Biometrics 1977;33:159-74

18. Bakshi R, Caruthers SD, Janardhan V, et al. Intraventricular CSF pulsation artifact on fast fluid-attenuated inversion-recovery MR images: analysis of 100 consecutive normal studies. AJNR Am J Neuroradiol 2000;21:503-08

19. Jack CR Jr, Rydberg CH, Krecke KN, et al. Mesial temporal sclerosis: diagnosis with fluid-attenuated inversion-recovery versus spin-echo MR imaging. $R a$ diology 1996;199:367-73

20. Hashemi RH, Bradley WG Jr, Chen DY, et al. Suspected multiple sclerosis: MR imaging with a thin-section fast FLAIR pulse sequence. Radiology 1995;196:505-10

21. Pipe JG. Motion correction with PROPELLER MRI: application to head motion and free-breathing cardiac imaging. Magn Reson Med 1999;42:963-69

22. Kidwell CS, Chalela JA, Saver JL, et al. Comparison of MRI and CT for detection of acute intracerebral hemorrhage. JAMA 2004;292:1823-30

23. Noguchi K, Seto H, Kamisaki Y, et al. Comparison of fluid-attenuated inversion-recovery MR imaging with CT in a simulated model of acute subarachnoid hemorrhage. AJNR Am J Neuroradiol 2000;21:923-27

24. Wiesmann M, Mayer TE, Yousry I, et al. Detection of hyperacute subarachnoid hemorrhage of the brain by using magnetic resonance imaging. J Neurosurg 2002;96:684-89

25. Fiebach JB, Schellinger PD, Geletneky K, et al. MRI in acute subarachnoid haemorrage: findings with a standardised stroke protocol. Neuroradiology 2004; $46: 44-48$

26. Sohn $\mathrm{CH}$, Baik SK, Lee HJ, et al. MR imaging of hyperacute subarachnoid and intraventricular hemorrhages at 3T: a preliminary report of gradient echo T2*-weighted sequences. AJNR Am J Neuroradiol 2005;26:662-65

27. Maeda M, Yagishita A, Yamamoto T, et al. Abnormal hyperintensity within the subarachnoid space evaluated by fluid-attenuated inversion-recovery MR imaging: a spectrum of central nervous system diseases. Eur Radiol 2003 13(suppl 4):L192-201

28. Essig M, Bock M. Contrast optimization of fluid-attenuated inversion-recovery (FLAIR) MR imaging in patients with high CSF blood or protein content. Magn Reson Med 2000;43:764-67

29. Anzai Y, Ishikawa M, Shaw DW, et al. Paramagnetic effect of supplemental oxygen on CSF hyperintensity on fluid-attenuated inversion recovery MR images. AJNR Am J Neuroradiol 2004;25:274-79

30. Dechambre SD, Duprez T, Grandin CB, et al. High signal in cerebrospinal fluid mimicking subarachnoid haemorrhage on FLAIR following acute stroke and intravenous contrast medium. Neuroradiology 2000;42:608-11

31. Filippi CG, Ulug AM, Lin D, et al. Hyperintense signal abnormality in sub arachnoid spaces and basal cisterns on MR images of children anesthetized with propofol: new fluid-attenuated inversion recovery finding. AJNR Am J Neuroradiol 2001;22:394-99 